\title{
Symptomatic Cerebral Ependymal Cyst : A Case Report
}

\author{
Rauf $P^{a}$, Aidil $M N^{a}$, Chan $K H^{b}$, Saufi $A^{b}$, Fadli $M^{c}$ \\ ${ }^{a}$ Department of Surgery, Hospital Tengku Ampuan Afzan, Malaysia \\ bUnit of Neurosurgery, International Islamic University of Malaysia IIUM, Malaysia \\ 'Department of Pathology, Hospital Tengku Afzan, Malaysia
}

\section{ABSTRACT}

Cerebral ependymal cyst is a rare benign neuroepithelial cyst. We report a case of cerebral ependymal cyst in a 62-year-old lady who presented with status epilepticus. She gave history of progressive right occipital headache over a year. Magnetic Resonance Imaging of the brain showed a large occipital cyst. She underwent a right craniotomy, deroofing of the cyst and insertion of Ommaya catheter. The clinicopathological aspects of the cyst are discussed.

KEYWORDS: Ependymal Cyst, Status Epilepticus, Cerebral, Headache

\section{INTRODUCTION}

Ependymal cyst is a rare benign neuroepithelial cyst. Typically, it presents in young adult with the mean age of 33 years with slight male predominance. ${ }^{1}$ The cyst wall is lined by ependymal cells. Most cysts are small and only become symptomatic when it becomes large. We present a case of symptomatic cerebral ependymal cyst in a 62 year old lady, presented with status epilepticus.

\section{CASE REPORT}

A 62 year old lady was presented with status epilepticus requiring intubation. She was extubated after three days of admission to the Intensive Care Unit. She gave a one year history of progressive right sided occipital headache. However, there was no history of seizure. On examination, there was left homonymous hemianopia with Grade II papilloedema. Other neurological examinations were unremarkable. Magnetic Resonance Imaging (MRI) demonstrated a $6.3 \times 5.5 \times 4.0 \mathrm{~cm}$ nonenhancing cystic mass at the right occipito-parietal

Corresponding Author:

Assoc. Prof. Datuk Dr. Mohamed Saufi Awang Senior Lecturer and Consultant Neurosurgeron Unit of Neurosurgery, Department of Surgery Kulliyyah of Medicine, International Islamic University Malaysia, Kuantan, Pahang, MALAYSIA

Email: saufiawang@iium.edu.my region (Figure 1). The patient underwent right occipital craniotomy. The cyst was opened, deroofed and the fluid was aspirated. A part of the cyst wall was biopsied. An Ommaya catheter was placed into the collapsed cyst. Post operatively, she was well with no seizure and total resolution of headache. Histopathology examination was reported as ependymal cyst (Figure 2). At follow-up appointments, she was asymptomatic and did not require any aspiration. No post operative scan was done as she was clinically monitored.

\section{DISCUSSION}

Ependymal cerebral cyst is a rare benign condition. It often presents in young adult with a slight male predominance. The cyst often occurs as an intracranial paraventricular cyst, commonly in the frontal or fronto-parietal region. ${ }^{2}$ Other cystic space occupying lesions which may resemble ependymal cyst are cystic glioma and arachnoid cyst.

The cyst most probably arises from the embryo-fetal period and remain silent. Although the exact mechanism is unknown, one theory suggested that the cyst arises from the ependymal rest that has become displaced into the cerebral substance and subarachnoid space during the early foetal development. The displacement may cause malformation of mante layer or pinching off the diverticulum, which result in an isolated ependymal cavity. ${ }^{3}$ Affected patients only become symptomatic 
when the lesion is large enough. Fluid will gradually accumulate in the epithelial cavity by several suggested mechanisms which includes active secretion, transcellular transport, and passive transport caused by fluid hyperosmolarity. ${ }^{4}$

Ependymal cyst can be a part of systemic disorders such as Aicardi Syndrome and orofasciaodigital syndrome. It may become space occupying, either by displacement of adjacent structures or by obstructing CSF circulation. Clinical signs are due to these effects or associated malformations as mentioned above.

Microscopically, ependymal cyst is lined by simple columnar or cuboidal cells, often ciliated, and resting on neuroglia. There is no fibrous capsule. It has positive histochemistry stain for $\mathrm{S} 100$ and GFAP. $^{5}$

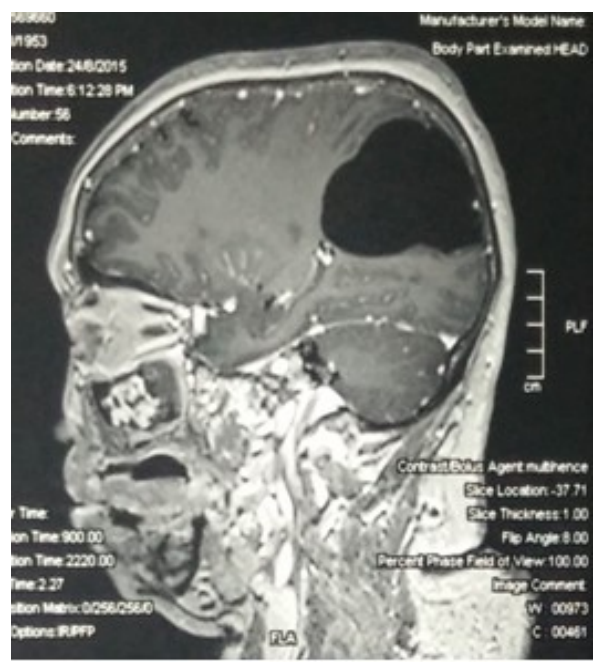

Figure 1: MRI showed non-enhancing well demarcated cystic lesion over the right parieto-occipital area.

There are various suggestions in term of the treatment over the years. Asymptomatic patient can be treated conservatively. Similarly, for symptomatic patients that responded well with medication. Meanwhile, surgery is recommended for symptomatic lesion that does not respond to medication, especially those with significant mass effect. ${ }^{6,7}$ Surgical approaches include simple stereotactic aspiration (repeated), subtotal excision or total excision whenever possible and/or shunt procedure. An endoscopic fenestration of the cyst to an adjacent CSF space is another option. ${ }^{4}$

The goal of the treatment is to establish a communication of the cyst with the subarachnoid space. Stereotactic aspiration is not practical due to the need of repeated therapy. Rarely, lesion is accessible for total excision to be carried out. Most of cases are treated with subtotal excision and deroofing of the cyst wall with creation of communication with the ventricle or subarachnoid space. In this case, we inserted Ommaya catheter after deroofing which will allow repeated aspiration if the cyst reoccurs.

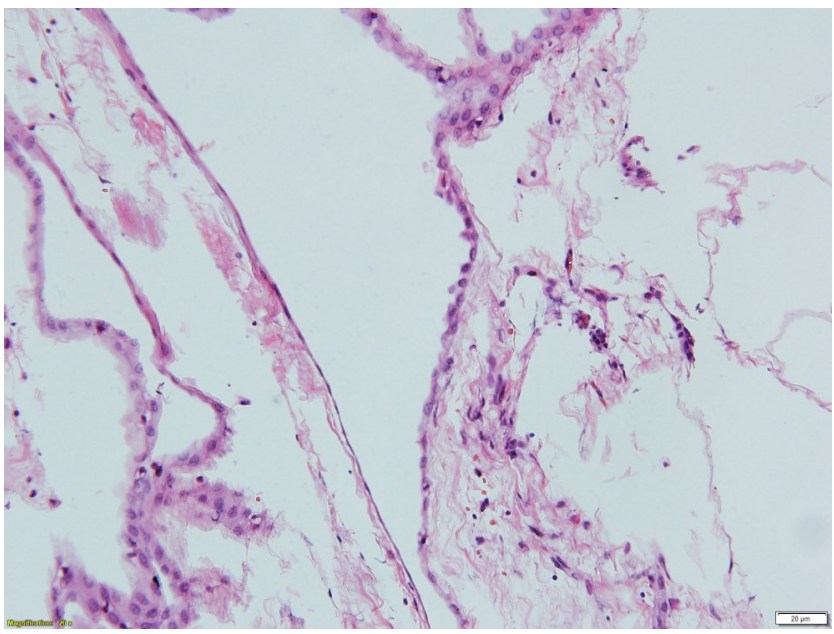

Figure 2: The H\&E stained slides show fragments of fibrous tissue lined by flattened to cuboidal epithelium having round nuclei and moderate amount of cytoplasm

\section{CONCLUSION}

Cerebral ependymal cyst is a rare condition. It usually becomes symptomatic when the cyst is large. Clinical manifestations vary from raised intracranial pressure symptoms like headache, vomiting, blurring of vision, seizure or as part of associated malformation. Depending on the symptomatology and size of the cyst, it can be treated conservatively or surgically with various options. 
There are various suggestions in term of the treatment over the years. Asymptomatic patient can be treated conservatively. Similarly, for symptomatic patients that responded well with medication. Meanwhile, surgery is recommended for symptomatic lesion that does not respond to medication, especially those with significant mass effect. $^{6,7}$ Surgical approaches include simple stereotactic aspiration (repeated), subtotal excision or total excision whenever possible and/or shunt procedure. An endoscopic fenestration of the cyst to an adjacent CSF space is another option. ${ }^{4}$ The goal of the treatment is to establish a communication of the cyst with the subarachnoid space. Stereotactic aspiration is not practical due to the need of repeated therapy. Rarely, lesion is accessible for total excision to be carried out. Most of cases are treated with subtotal excision and deroofing of the cyst wall with creation of communication with the ventricle or subarachnoid space. In this case, we inserted Ommaya catheter after deroofing which will allow repeated aspiration if the cyst reoccurs.

\section{REFERENCES}

1. H Nakase, $\mathrm{Y}$ Ishida, $\mathrm{T}$ Tada et al.

Neuroepithelial cyst of the lateral ventricle:

Clinical features and treatment. Surg Neurol. 1992;37:94-100.

2. RL Friede and MG Yasargil. Supratentorial intracerebral epithelial (ependymal) cyst: review, case report, and fine structure. J Neurol Neurosurg Psychiatry. 1977;40:127-137.

3. DC Bouch, I Mitchell and AJF Maloney. Ependymal lines paraventricular cerebral cysts: A report of three cases. J Neurol Neurosurg Psychiatry. 1973;36:611-617.

4. B Pant, T Uozumi, T Hirota et al. Endoscopic resection of intraventricular ependymal cyst presenting with psychosis. Surg Neurol. 1996;46:573-578.

5. S Coca, A Martinez, J Vaquero, M Moreno et al. Immunohistochemistry study of intracranial cysts. Histol Histopathol.1993;8:651-654.

6. T Marioka, S Nishio, S Suzuki et al. Choroidal tissue cyst in the temporal horn associated with complex partial seizure. Clin Neurol Neurosurg. 1994;96:164-167.

7. A Guermazi, Y Miaux, JF Majoulet et al. Imaging findings of central nervous system neuroepithelial cysts. Eur Radiol. 1998;8:618-623 
